\title{
3D INSPECTION OF THE RESTORATION AND CONSERVATION OF STAINED GLASS WINDOWS USING HIGH RESOLUTION STRUCTURED LIGHT SCANNING
}

\author{
M. Rahrig ${ }^{1, *}$ M. Torge ${ }^{2}$ \\ ${ }^{1}$ KDWT, Centre for Heritage Conservation Studies and Technologies, University of Bamberg, Germany \\ max.rahrig@uni-bamberg.de \\ ${ }^{2}$ Bundesanstalt für Materialforschung und Prüfung (BAM), D4.5 Analysis of Artefacts and Cultural Assets, Berlin, Germany \\ manfred.torge@bam.de
}

Commission II, WG II/8

KEY WORDS: stained glass, restoration and conservation, structured light scanning, 3D inspection, glass scanning

\begin{abstract}
:
The initial focus of this research was on the development of a general workflow for the documentation and monitoring of historical stained glass windows using structured light scanning. Therefore windows from different churches, time periods and with different corrosion and damage phenomena were scanned before and after conservation measures. To evaluate the execution of the restoration measures the data was compared using 3D inspection software to examine the differences in geometry between the two scans. Various problems had to be solved, for example, how to deal with heavily reflective surfaces and the extreme contrast between light and dark surfaces, as seen in the borders between 'Schwarzlot' painting and plain glass. The application of materials for matting the surfaces, such as Cyclododecane spray, was impossible due to the high accuracy of the surface measurement required for 3D inspection. Regarding the contrast differences of the surfaces, the creation of exposure fusions and the use of polarization filters to reduce reflections were tested. In addition to the general problems encountered when recording translucent surfaces, the historical glasses caused additional problems in calculating surface comparisons. For example, the windows have to be moved and turned around several times, both during the conservation process and while scanning, causing deformations of the geometry due to the flexible lead rods allowing a certain degree of movement.
\end{abstract}

\section{INTRODUCTION}

As part of a research project for the training and qualification of Polish specialists in the restoration of glass paintings, funded by the 'German Federal Environmental Foundation' (DBU) and the 'Deutsch-Polnische Stiftung Kulturpflege und Denkmalschutz' (DPS) with funds from the Federal Republic of Germany, and in cooperation with the 'Bundesanstalt für Materialforschung und prüfung' (BAM) and the KDWT/ University of Bamberg, highresolution $3 \mathrm{D}$ documentation was used to evaluate the execution of the restoration measures. For this purpose, historical stained glass windows were recorded using structured light scanning (SLS) before and after the conservation treatment. The data was then analysed using 3D inspection software in order to examine the differences in geometry between the two scanning campaigns.

The windows are from three churches in Poland, and different time periods; the medieval ones are located in Koszewko, the glasses from the early $20^{\text {th }}$ century in Legnica, and the glasses from Oswiecim date back to the mid- $20^{\text {th }}$ century. They all exhibit different corrosion and damage phenomena.

\subsection{The windows from Koszewko}

In the village church of Koszewko, stained glass paintings showing the coats of arms of the 'von Küssow' family, dating back to the $15^{\text {th }}$ century, have been preserved (Fig 1). They display environmental corrosion damage and loss of the painting layer as well as glass breakage and defects in the lead rods. In the course of their restoration, areas with darkening were brightened by manual cleaning. The brightening process causes particular difficulties in the 3D recording after restoration, as it leads to increased reflection.

\subsection{The windows from Legnica}

The windows from the Liebfrauenkirche in Legnica (Fig 1) have high-quality glass paintings dating from 1905/6. They were manufactured in various well-known German glass workshops: Franke, Naumburg; Müller, Quedlinburg; Geiges, Freiburg; Oetken, Oldenburg; Linnemann, Frankfurt/M. Due to the lack of protective glazing, the 'Schwarzlot' paintings are at risk and suffer particularly from flaking. 'Schwarzlot' is the colour used for stained glass and consists of glass particles and metal compounds burnt into the glass surface after application (Brepohl, 2013). Therefore the main focus of the conservation was a careful cleaning of the glasses and consolidation of the paintings. The 3D inspection was carried out to determine whether the removal of the dirt layers is measurable.

\subsection{The windows from Oswiecim}

The stained glass windows in the parish church of Oswiecim (Fig 1) date back to 1940 . They were produced by Müller, Quedlinburg. Due to thermal stress, presumably caused by fire, the glass has a very strong craquelé, many cracks and flaws, and the paint is deteriorating. During conservation, the complex crack pattern was strengthened with acrylic, and the $3 \mathrm{D}$ inspection was used to check whether the acrylic resin is only inside the cracks or has been additionally deposited as a layer on the surface.

\footnotetext{
* Corresponding author
} 


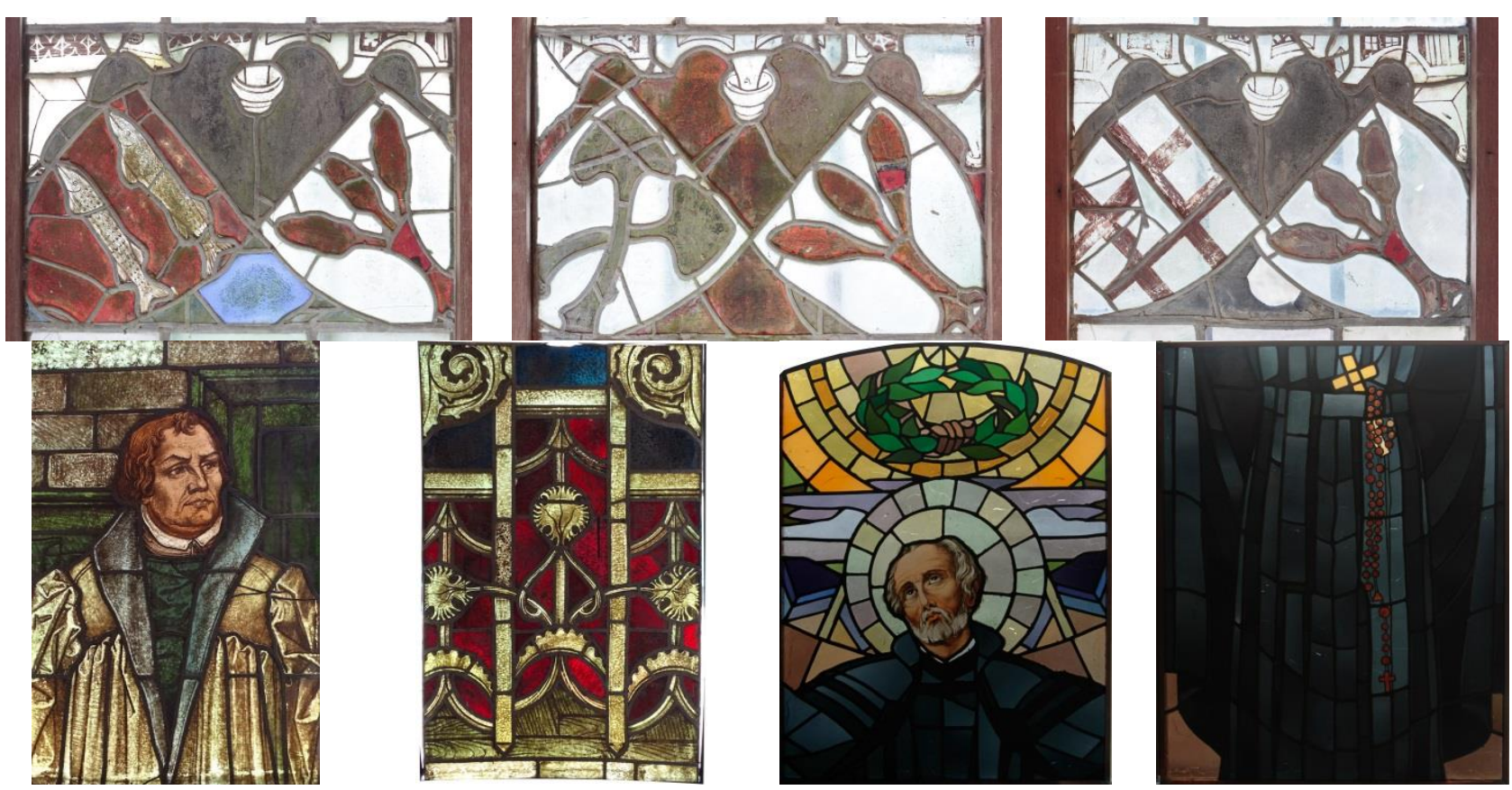

Figure 1. Overview of the Polish stained glass windows. In the upper line from left to right: Koszewko $n I I, n I I I$, sIII. In the bottom line from left to right: Legnica $n x 3 b, n x 4 b$ and Oswiecim $3 b$ and $2 b$. (Oleszczuk 2017-2019)

\section{TECHNICAL BACKGROUND}

The three-dimensional measurement of glass surfaces is a relatively complex problem. Common sensors used for noncontact measurement of surfaces are based predominantly on two basic techniques; active and passive systems. Active sensors include techniques that apply an impulse to the surface to be documented and then detect its reflection. The impulse can be a laser beam or a light pattern, so this category includes both laser and structured light scanners. In passive systems, the surface is not stimulated; the sensors detect the surface on the basis of shape from shading, shape from motion and comparable methods. Passive systems include, for example, stereo cameras, but also the calculation of image groups from individual images; both based on Structure from Motion (SfM) and Image based Modeling (IbM). In both cases, the surface of the object to be documented must be defined clearly, i.e. it should be opaque and not translucent (Stylianidis et al., 2016).

Glass, on the other hand, is characterised by its optical properties as a translucent material. Due to its translucency, the glass surface is difficult to capture optically, which considerably impairs the common systems used for 3D measurement. Over the past several years, various systems have been developed to solve this problem (Eren, 2010; Ihrke et al., 2010; Yeung, 2011; Mériaudeau et al., 2012). These are, for example, processes that stimulate the surface thermally or with UV light (Rantson et al., 2010), while other systems make use of the light refraction properties of the glass object in a bath of fluorescent liquid (Hullin et al., 2008). All these procedures share two fundamental problems. The devices are usually custom-made or prototypes and therefore not freely available on the market, and they have extremely limited usability in the context of the documentation of cultural heritage. It is usually impossible to either thermally stimulate fragile historical objects, or immerse them in liquid.

In various projects undertaken in the field of preservation sciences at the University of Bamberg, it has been observed that corrosion phenomena alter the optical properties of historical glass paintings in a way that is detectable using high-resolution, three-dimensional measurement with conventional structured light scanners (Drewello, Kleine et al., 2010; Drewello, Wetter et al., 2010; Drewello, Kleine et al., 2016). Due to the centuries of exposure to environmental phenomena weathering crusts, lime, gypsum and dust deposits form on the windows, result in a wafer-thin, opaque layer on the surface. This layer can be used to measure the surface with optical systems. Salemi et al. achieved similar results using a Konica Minolta Laser Scanner (Salemi et al., 2008).

In structured light scanning, a projector transmits a pattern of light onto the surface to be measured; the deformation of the pattern on the surface is then captured by a camera. In this way, 3D coordinates can be calculated (Rahrig et al., 2018; Gühring, 2002; Akce, 2007; Floth et al., 2011). The scanner manufacturers generally recommend that the scanner is placed as straight as possible, orthogonal to the surface to be measured - this ensures that the largest possible area can be captured within a single scan, and the surface reflects the light of the projector optimally to the camera. In this way opaque surfaces can be captured with comparatively short exposure times for single scans. In the case of glass surfaces, however, this setup is problematic; strongly reflecting spots are created, leading to an overexposure of the scan and thus holes in the data. If, however, the scanner is placed at an angle between $80^{\circ}$ and $45^{\circ}$ to the surface to be documented, the highlights and specular reflections are not reflected directly into the camera, avoiding overexposure. The drawback with this setup is that the whole field of view of the scanner is not used optimally and therefore the area recorded is a little smaller. However, the data recorded in this area are more homogeneous so the overlaps between individual scans can be reduced and fewer additional scans are needed to fill holes caused by reflective hotspots.

Another problem is that the heterogeneous thickness and colour of the corrosion and dirt deposits adjacent to non-corroded surfaces, as well as glossy lead rods and both dark and light 'Schwarzlot' paintings, require very different exposure settings in order to document the glass as completely as possible. Dark areas require longer exposure times, bright surfaces shorter. Therefore a combined scan of the glass surface with different exposure settings can be helpful. With the device used, the Comet L3D from Steinbichler, it is possible to create a so-called 
exposure fusion analogous to an HDR photo. Here, two or three recordings with different exposure settings are merged into one single scan, which thus contains better surface information over the entire area captured.

\subsection{Cyclododecane for matting the glass surface}

The use of matting sprays, which are frequently used in industry for scanning glossy surfaces, is problematic in the field of heritage preservation. In general the components of the sprays are not fully known and thus potentially contain solvents which could react negatively with the historical objects and materials. In addition, in order to remove the sprays after scanning, a mechanical cleaning of the surfaces is required; which could lead to additional damage. Only Cyclododecane, a volatile binder that has long been used by restorers for temporary consolidation, can be used as a matting spray (Díaz-Marín et al., 2015). As it sublimates residue-free within a few hours, no mechanical cleaning is necessary and, with the exception of some modern plastics, it does not react with materials. Cyclododecan has been used at the University of Bamberg for matting surfaces since 2005 (Bellendorf, 2007). However, experience has shown that the available sprays do not usually apply a uniformly thin film, but tend to form (small) lumps. The use of Cyclododecane had to be dispensed with in the case of the stained glasses documented here as the uneven distribution and layer thickness would falsify the results of the 3D comparison of the condition before and after the cleaning and preservation measures.

\subsection{Polarization filters to reduce reflections}

Compared to the glass paintings from Koszewko, Straubing (Drewello, Kleine et al., 2010 and 2016) and Erfurt (Drewello, Wetter et al., 2010), the glasses from Legnica and Oswiecim are relatively young, dating back only as far as the beginning and first half of the $20^{\text {th }}$ century. Due to technical developments and advancing industrialisation, the raw materials for glass production were much purer by that time, making it possible to produce higher quality and more stable glasses (Wedepohl, 2003). In addition, these glasses have been exposed to the weather for a far shorter time than the medieval examples. Thus these glasses are in a better condition; their luminosity is more intense, but the corrosion crusts on the surfaces are less pronounced. Therefore, scanning was expected to produce significantly stronger reflections. In order to reduce the mirror effects of these young stained glasses, the use of polarization filters was tested.

Light sources such as LED, light bulbs and even the sun emit light rays in disordered waves. When these light rays are reflected by a surface, for example water, the beam is fanned out and emitted in different directions as scattered light. Polarising filters have the property of only allowing light with a single wave orientation to pass through, with other wave orientations being blocked (Chen et al., 2007). Three setups were tested for the documentation of the stained glasses: A polarising filter in front of either the projector or camera, and a combination of both. The filter in front of the projector should reduce the reflections on the glass surface, while the filter in front of the camera should filter the light to the sensor, reducing the scattered light reflected by the glass surface. Chen et al. has already shown the possibilities of polarising filters for scanning translucent objects and has achieved good results for marble, fruits and a modern glass vase (Chen et al., 2007).

The empirical studies in this project, using filters to scan the glass paintings were unfortunately not that successful. With the use of two polarising filters, one in front of the projector and one in front of the camera, not enough light was transmitted to the image sensor using either crossed or parallel filters, making data acquisition impossible. Using a single polarising filter in front of the projector or in front of the camera produced similar results. In both cases the reflections were reduced, but the filters blocked too much light. Thus, the exposure time and light intensity of the projector had to be increased to a degree impossible with the highest possible light settings supported by the scanner used (Fig 2). The best results for scanning the glass surfaces were therefore achieved without any filters, and with the scanner positioned at an angle of $80^{\circ}$ to $45^{\circ}$ to the object.

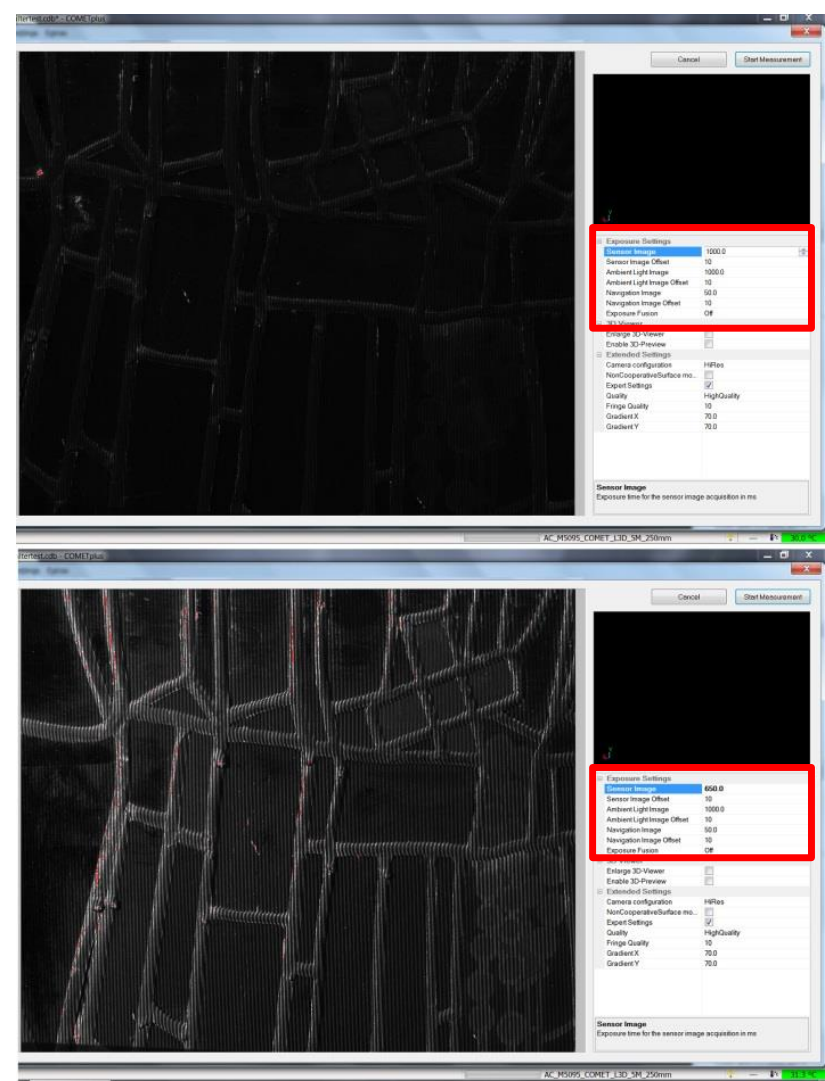

Figure 2. Oswiecim 2b; Comparison of different light setups, within the scanner software, with and without the use of polarising filters. Upper part: Setup with filter; highest possible light settings don't provide enough light for scanning. Lower part: without filter, but scanner located $70^{\circ}$ to surface; ideal light settings.

\subsection{Data processing}

The aim of the 3D documentation of the stained glass paintings was a comparison of the surfaces before and after the restoration. The very small differences between the two measured surfaces required the data to be analysed extremely carefully. For example, the structured light scanner was calibrated according to the manufacturer's specifications at regular intervals and after every transport and change of lenses. After completion of the measurements, an iterative-closest point matching (ICP/'best-fit') between individual scans was carried out to ensure the best possible alignment. Once the point cloud scans were converted into a surface model, the resolution of the mesh was adapted to the resolution of the lenses used; $100 \mu \mathrm{m}$ for the $250 \mathrm{~mm}$ lens which captured both the front and back of the windows in their entirety; and $45 \mu \mathrm{m}$ for the $75 \mathrm{~mm}$ lens used for partial areas of a few individual glass panes per window. 
The data sets were neither smoothed nor were holes interpolated and closed. If some individual glass panes had particularly strong reflections, it was checked during post-processing whether the glass surface had been captured adequately, or whether the data was noisy, and the noisy areas were removed manually. The overview of the windows is therefore partially incomplete, but this is preferable to inserting interpolated data which would falsify the surface comparisons. The postprocessing of the data was carried out using the COMETplus v.9.91 software by Steinbichler, and Geomagic Wrap 2017. The data were saved as STL-files and imported into GOM Inspect 2018 for the calculation of the 3D comparisons.

\section{GENERAL RESULTS OF THE SINGLE CAMPAIGNS}

The high-resolution documentation of stained glasses in individual campaigns has already shown potential in providing informative surface observations. As already demonstrated by Drewello, Kleine et al. with the example of the medieval Moses window at St Jacob in Straubing, the stained glass can be recorded accurately with the help of structured light scanning. Due to the difference in topography, not only can 'Schwarzlot'painting be separated from the glass surface, but statements on the painting technique can also be made. For example scanning can reveal which tools were used to apply the 'Schwarzlot' or how luminous accents were set. Furthermore the 3D surface documentation can help in the reconstruction and retouching of faded areas (Drewello, Kleine et al., 2010 and 2016).

'Schwarzlot' is a colour that has been used for glass painting since the Middle Ages. It consists of a mixture of finely ground glass powder, carbon, metal and a variety of other components. The viscous paint is applied to the glass panes, and can be drawn on using brushes, or as a thin layer with highlights and details scratched in using soft tools such as wooden sticks. After drying, the glasses are heated, whereby the glass particles of the 'Schwarzlot' form a stable and long-lasting bond with the glass surface (Brepohl, 2012).

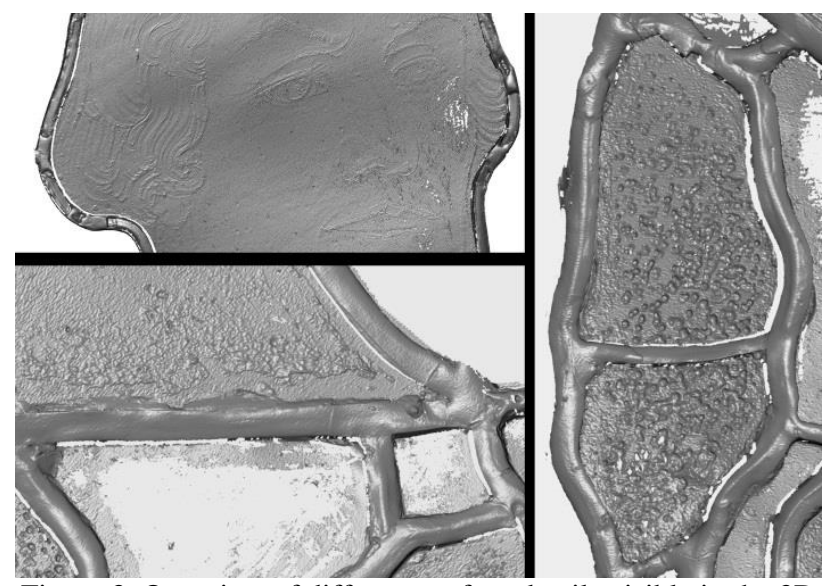

Figure 3. Overview of different surface details visible in the 3D scan; top left: 'Schwarzlot' painting of a face; bottom left: dust and dirt layers on the glass surface, next to clean glass panes; right: strongly corroded glass pane with a repair lead rod in the middle.

The paintings of the stained-glass windows in Koszewko and Legnica are perfectly visible in the 3D model (Fig 3, top left). Further surface information can be found in the 3D models, for example the effects of different corrosion phenomena. These can be weathering crusts and dust and dirt layers that are deposited on the glass surface (Fig 3, bottom left), but also corrosions that decompose the glass surface piece by piece and leave a craterlike landscape in the glass (Fig 3, right). Cracks in the glass and historical repairs, such as those using lead rods, can also be detected (Fig 3, right). Area in which the glass surface is in an excellent condition, are represented in the 3D scan as negative findings, without surface information (Fig 3, down left). Due to the lack of weathering crusts and other deposits, this glass is not opaque but still completely translucent, making measurement of the surface impossible.

\section{3D INSPECTIONS}

\subsection{D Inspections of the whole window}

The main aim for the 3D documentation of the historical stained glasses was to compare the surfaces before and after conservation. An initial juxtaposition of the two data sets for a visual comparison provides at first only limited information. It can be seen clearly that in some areas the cleaning of the surfaces has significantly increased the light transmittance, and that the scans from after the conservation have relatively more holes or missing parts due to areas that cannot be scanned (Fig 4). However, further differences between the two campaigns can be detected only to a very limited extent in this way. A mathematical comparison of the models by means of 3D inspection software is therefore used to objectively record changes.
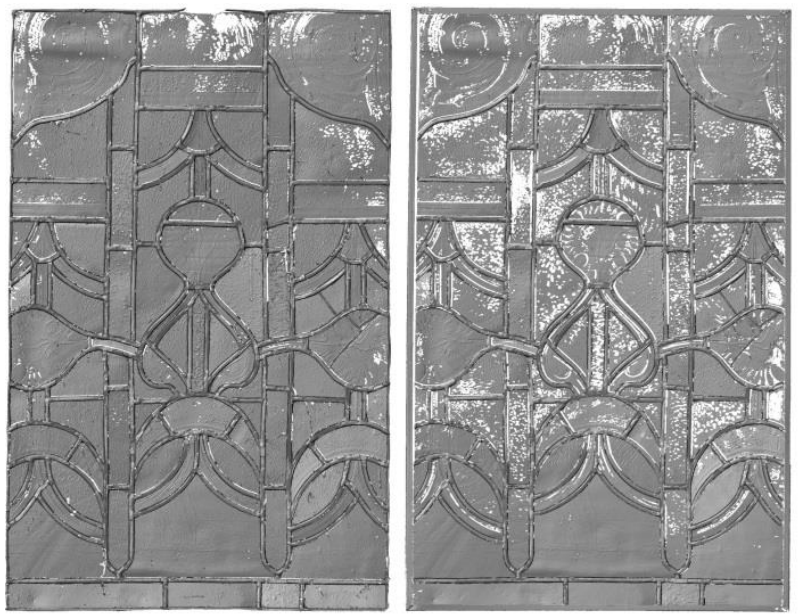

Figure 4. Juxtaposition of window $n X 4 b$ from Koszewko before (left) and after the conservation (right).

GOMinspect 2018 software was used to calculate the comparisons. For this purpose, the STL data of the initial state was loaded as the reference model and the second model from after conservation and cleaning as the so-called actual model. In these comparisons, the geometric differences between the two 3D models are visualised in a false colour image. Blue areas show shrinkage of the surface and yellow and red areas an increase (Fig 5).

Due to the work processes in the conservation environment, it was not possible to set up a static reference system for the orientation of the scans between the different campaigns; the glasses had to be turned around several times during their conservation in order to work on the front and back sides. Additionally both sides were documented photographically on multiple occassions. Due to these many transports and movements within the restoration workshop, the 3D models of the glasses had to be aligned to each other digitally by means of an iterative-closest point (ICP) or 'best-fit' method. The glasses were first aligned roughly by hand using a three-point prealignment, after which the ICP could be calculated. 
The standard deviation of the scans using the $250 \mathrm{~mm}$ lens in a resolution of $0.1 \mathrm{~mm}$ is disproportionately large after alignment, on average about $0.8 \mathrm{~mm}$. A closer look into the surface comparison shows the reason for this (Fig 5). The large deviations between the 3D data from the initial condition and after conservation do not result from the conservation measures themselves, but rather from the freedom of movement of the lead rods. Since lead is not a rigid metal but soft, slight movements of the lead rods occur during the handling of the windows. The middle part of the glass in particular tends to sag slightly, which leads to bowl-shaped deviations in the 3D comparison. Similar effects have already been experienced with the medieval windows from Erfurt (Drewello, Wetter et al., 2010). For the 3D comparisons, this movement is counterproductive, but for the historical glass windows it is an important protective factor. This flexibility, for example, compensates for wind or storm gusts which can press against the windows, thus preventing the individual glass panes from breaking.

Nevertheless, certain changes, for example the reinforcements on the edges of the stained glass windows, can be seen in the overall view of the panes, Here, copper sheet frames were placed around the windows during conservation to give them more stability, making it easier to reinstall them in the church. Changes in the lead net can also be recorded (Fig 5). These may be areas where lead rods were exchanged, i.e. where new rods were added. However, there are also places where lead rods used for repair have been removed, as cracked glasses that were secured with a lead rod during a previous historical restoration were now glued during the modern restoration.

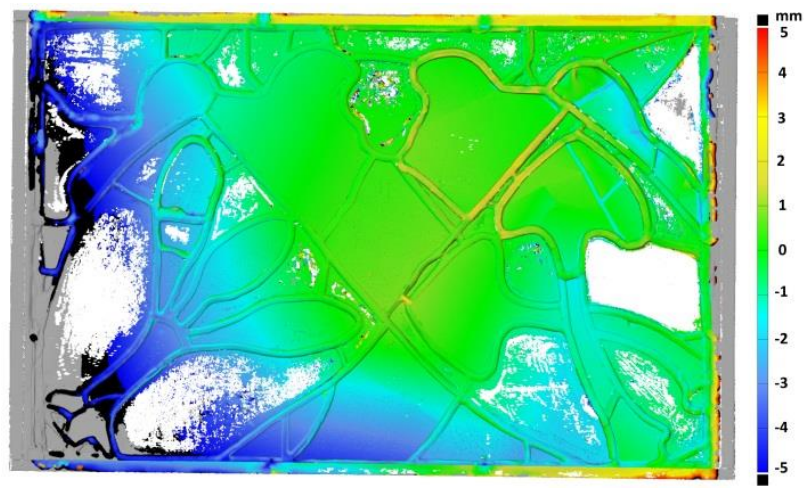

Figure 5. 3D inspection of the outside of the stained glass window $n I I I$ from Koszewko before and after its conservation.

\subsection{D inspections of single glass panes}

Since the corrosion layers are thin on the historical glass surface, in addition to the scans of the entire windows, single individual glass panes were documented with a higher resolution. A $75 \mathrm{~mm}$ lens with a resolution up to $45 \mu \mathrm{m}$ was used for this purpose. This lens captures a much smaller area per single scan than the $250 \mathrm{~mm}$ lens, with a field of view of about 5 $\mathrm{x} 8 \mathrm{~cm}$, compared to $18 \times 25 \mathrm{~cm}$ with the $250 \mathrm{~mm}$ lens. With a constant image sensor of 5 megapixels inside the scanner, the surface resolution increases from 100 to $45 \mu \mathrm{m}$. For each church and documentation campaign, a selection of individual glass panes to be documented was made in consultation with the restorers. Glass panes were selected specifically to show representative damage phenomena for the respective windows. During scanning the surrounding lead rods were also recorded in order to detect changes both on the glass surface and on the rods themselves.
The scans were processed in the same way as the scans of the entire windows, and the alignment of the scans in GOMinspect 2018 also carried out using ICP. However, only the surface of the glass, without lead rods, was used to calculate the alignment. This selection-based alignment serves to calculate the $3 \mathrm{D}$ comparisons much more exactly, as it ignores the movement of the lead rods.

The restoration measures for the Polish glasses were not carried out as a comprehensive removal of entire layers; instead, individual measures were taken at corroded areas. It was therefore expected that the geometry of a single glass pane would not change significantly. Deviations and falsifications of the 3D comparisons due to slippage of the individual glass panes between movable lead rods are also counteracted by the selection-based 'best-fit'. The standard deviation for the alignment of the scans between the two campaigns was thus about $0.05 \mathrm{~mm}$ on average.

4.2.1 Koszewko: Information regarding several different conservation measures can be captured in the high-resolution 3D inspection of the individual glass panes. Figure 6 shows the 3D comparison of the inside and outside of a single pane from a coat of arms representation from window nII from Koszewko. The pane shows one of the two fish (pikes) from the coat of arms. Since the glass pane was broken in the middle, the alignment was only calculated on the front part of the fish, and. five conservation treatments can be clearly identified. When looking at the outside surface, it can be seen that the left part shows extreme changes. They extend from more than $-1 \mathrm{~mm}$ in the lower area of the glass pane up to $+0.6 \mathrm{~mm}$ in the upper area. Here, it is clear that movement of one of the glass panes took place. The movement can be traced back to the gluing of the two divided glass panes together. After conservation, they form a uniform plane surface.

It was necessary to first remove the two glass pieces in order to glue them together. To do this, the lead rods had to be carefully removed, resulting in deformations. The lower rod was merely bent up, resulting in changes of up to $+/-1 \mathrm{~mm}$ in the shape of the lead. The upper rod, on the other hand, was replaced, resulting in significantly greater changes in the surface.

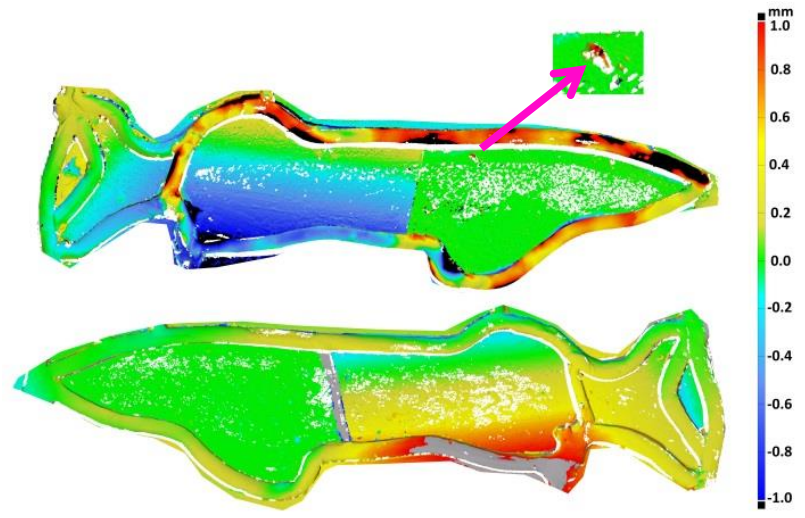

Figure 6. 3D inspection of a single glass pane from $n I I$, Koszewko. Top: comparison of the outside; bottom: comparison of the inside.

The comparison of the inner side of the window also shows a significant change in the transition between the two partial glasses. During the restoration a repair lead that was no longer necessary, was removed after the glass panes were reassembled. Another anomaly on the surface can be detected: On the right part of the glass pane (Fig 6, detail), near the upper lead rod, 
there is an area in which an increase of the surface compared to the initial state is visible. This is most likely leftover window putty. After the glass pane had been reassembled and inserted into the lead net, the space between the glass pane and the lead rod was filled up with putty, creating a stronger connection between the glass and the surrounding leads, thereby increasing the stability of the windows. This also prevents the individual glass panes from slipping and falling out.

The white areas are areas that could not be scanned during the second campaign. Here, after conservation, the light transmission of the glass was too strong to allow the detection of the surface.

In another single glass pane it can be clearly seen how carefully and cautiously the conservation was carried out. When looking at the entire pane, hardly any changes are noticeable, so a part of the image has been enlarged (Fig 7). The partial area shows a section from the outside of the window. Logically, the exterior areas are more exposed to the weather, which is why stronger corrosion products are to be assumed. The detail shows the surface of the glass with various small structures. These are the edges of the weathering crust. The $3 \mathrm{D}$ inspection shows that only a very few loose areas of the crust have been removed. The other areas seem to have a stable connection with the glass, so that an overall removal of the corrosion layer was not necessary and might have damaged the glass. The black areas are due to small holes in the initial condition scan, and in the left corner of the detail, a small particle of window putty is again visible.

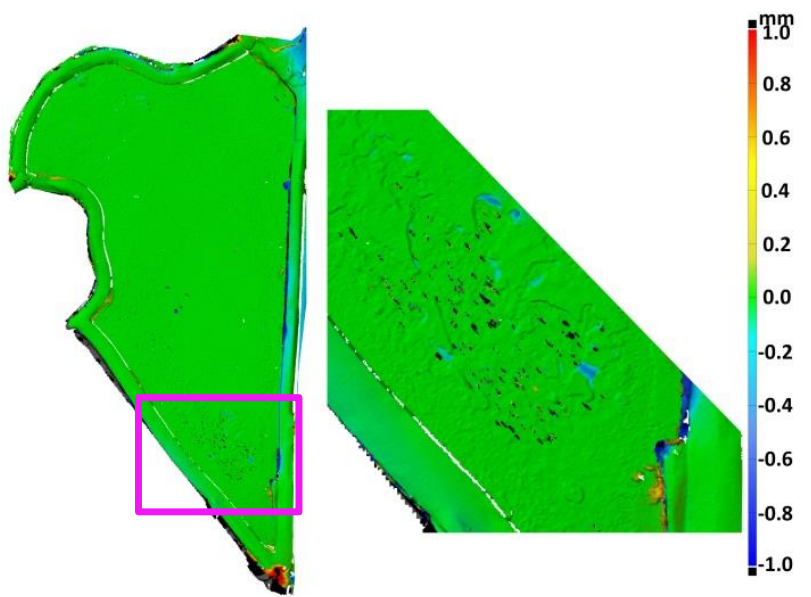

Figure 7. Overview and detail of the 3D inspection of a single glass pane from $n I I$, Koszewko.

The window nIII from Koszewko was in a poor condition before conservation (Fig 5). Various glass panes were broken, and some fractures were already fixed by repair leads. In the course of conservation, the repair lead was removed and, as the detail section shows (Fig 8), the highly fragmented panes were reunited with great care. No residues of the adhesive are visible on the glass surfaces, demonstrating that the acrylic resin (Araldit $2020^{\odot}$ ) was applied very carefully to the edges of the fractured fragments without running over the surfaces.

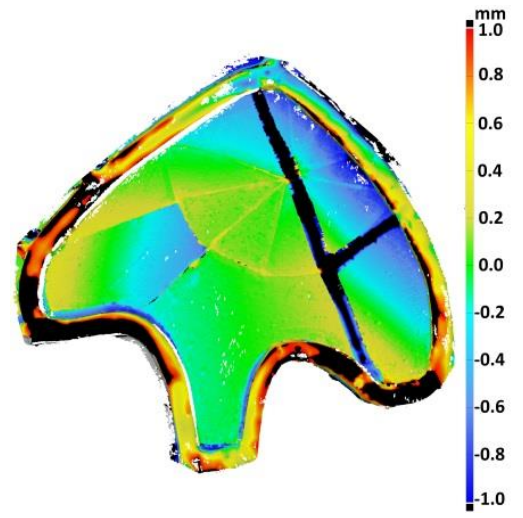

Figure 8. 3D inspection of $n I I I$, Koszewko, showing the restoration of strongly fragmented glass panes.

4.2.2 Legnica: The surface comparison of the inside of the portrait of Martin Luther in window $n X 3 b$ of the church of Legnica shows no measurable changes (Fig 9). Here the 'Schwarzlot' painting was consolidated, and the works left no residue on the surface. The scans of a single campaign clearly reveal the filigree line drawings of the face (Fig 3, top left). In comparison to the transmitted light photo, strong brightness gradients in the glass are visible, but it is only one single glass pane and not a composition of several different coloured panes. These colour differences are instead due to different glass thicknesses. On the reverse side a clear step can be seen in the glass (Fig 10). Here, during the production of the glass painting, a part of the surface was etched off, resulting in a different refraction behavior of the glass.

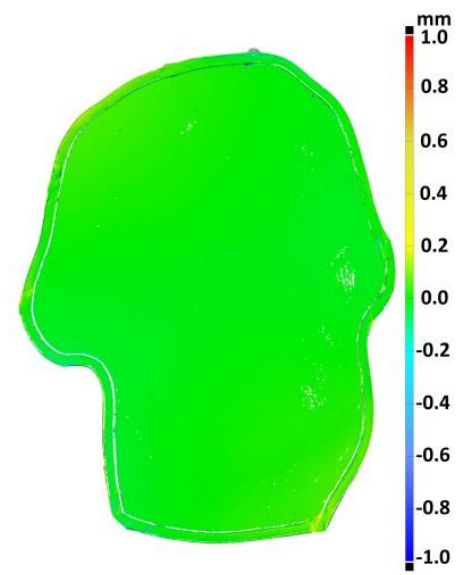

Figure 9. 3D inspection of a glass pane of $n X I 3 b$ from Legnica.
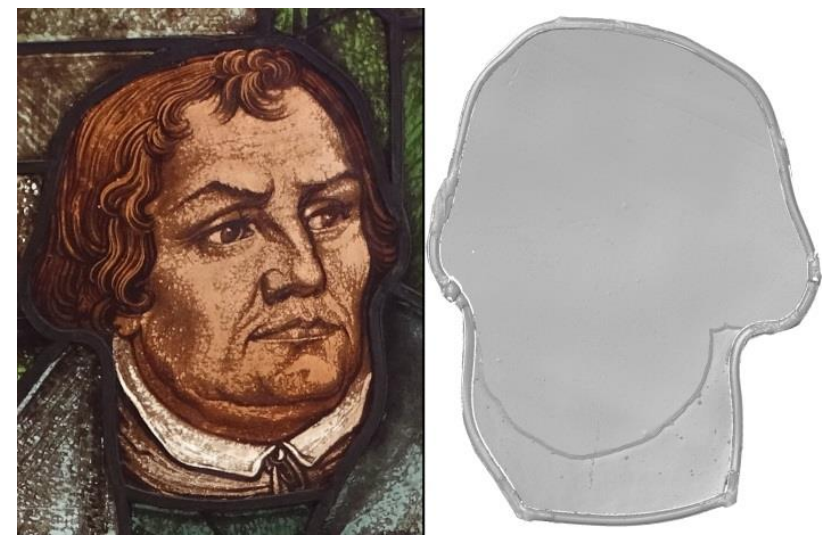

Figure 10. Left: transmitted light image of the glass pane from $n X I 3 b$ (Oleszczuk 2019). Right: view of the outside (3D scan). 
4.2.3 Oswiecim: In the 3D scans of the Oswiecim windows, the extreme damages of the craqueled glass panes are clearly visible in the overall view (Fig 11, 12). The cracks are spread chaotically through the panes, but the representation of the inner side with the various missing parts in the scan also shows that the glasses are otherwise in a relatively good condition. They are much more translucent than the medieval windows from Koszewko.

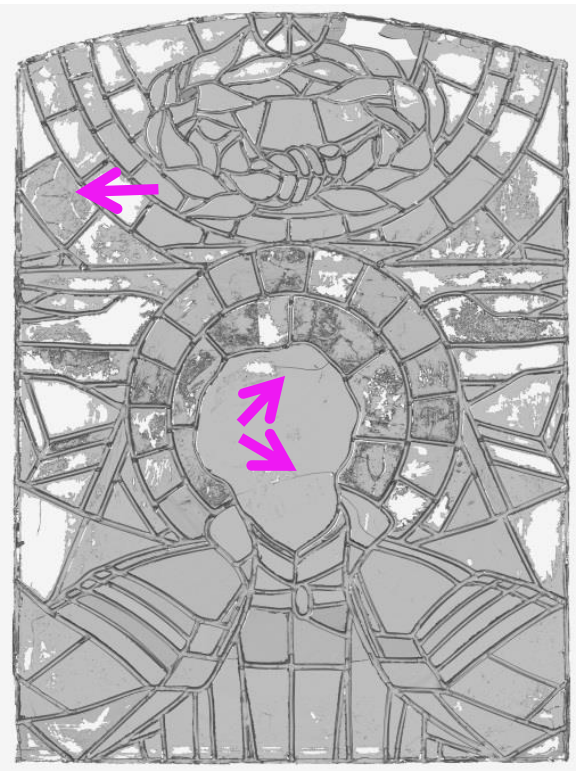

Figure 11. Overview of window $3 b$ from Oswiecim before conservation (inside). Some cracks are marked pink.

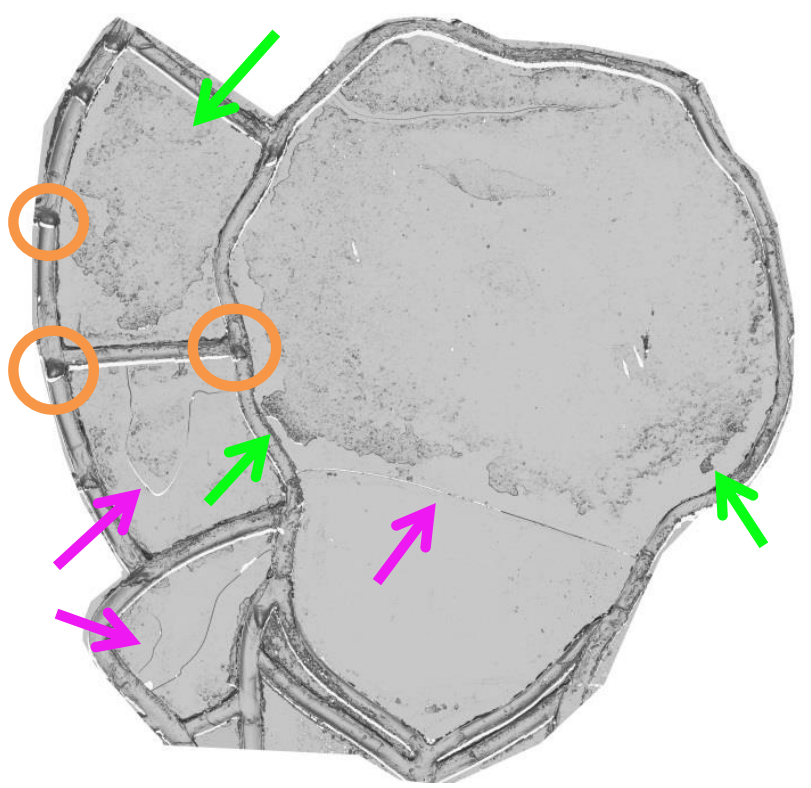

Figure 12. Detail of window $2 b$ from Oswiecim (outside).

Orange: The solder drops caused strong heat; Green: the thin lime slurry; pink: craqueled glass panes

The detailed examination of the high-resolution scans provides further information on the reason for the cracks. At the junctions between the individual lead rods, drops of solder are located on both the inside and outside (Fig 12). The drops are all on the lower edge of the solder joints, and therefore seem to have formed only when the windows were already installed upright in the church. If the droplets had instead formed when the stained glasses were leaded in the glazier's workshop, there would have to be differently oriented droplets as the windows would have been processed lying horizontally on a table. It can therefore be assumed that the windows were exposed to a strong source of heat at some point during their time in the church, presumably from a fire. However, no fire is recorded in the church itself since the glass was installed in the 1940s and therefore it is possible that it must instead have taken place outside, in the immediate vicinity of the windows.

On the outside of the Oswiecim windows, extremely uneven structures are clearly visible in the 3D scan (Fig 11). Analysis has shown that this is gypsum slurry applied over large areas of the windows, leading to strong darkening of the glass. During restoration, this lime layer has to be removed in order to restore the original luminosity of the glasses. Unfortunately, the documentation of the conservation is still pending, which is why no 3D inspection of the Oswiecim windows can be presented here.

\section{CONCLUSION}

Within the research project it was shown that the threedimensional documentation of historical (painted) stained glass windows with a commercial structured light scanner is possible. Scanning can be done without any special constructions, modifications of the scanner or coating of the historical objects. The high-resolution observation of the glass can provide useful information regarding the production of the glass painting and on damage and corrosion phenomena.

Large-scale calculation of 3D surface comparisons of historical stained glass before and after conservation, or for monitoring of weathering is, however, problematic, as the flexible lead net offers too much scope for movement. When installed in the churches, this freedom of movement is required as a shock absorber against strong wind loads, and during work in conservation workshops, free access to the front and rear sides of the windows must be ensured for a smooth workflow. For these practical reasons, it is therefore not possible to install a fixed referencing system on the windows.

Therefore, only extreme changes or interventions can be detected in the comparisons of the entire windows. For example, changes in the lead rods and auxiliary constructions such as reinforcements at the edges of the windows can be detected. In addition, the change in the light transmission behavior of the glass can be measured.

Observations of 3D inspections of single glass panes, however, provide impressive results, revealing details about the conservation process. Here, not only small changes to the lead rods, but also the careful restoration measures conducted on the glass surfaces can be documented in high resolution. The removal of corrosion products, the bonding of cracks as well as traces of window putty from fixing the individual panes in the lead net can be recorded.

Since the 3D measurement and calculation of surface comparisons in the context of the conservation of historical stained glass is comparatively complex, it can be assumed that such documentation is not applicable to the entirety of a medieval church's windows. Rather, this method should be carried out specifically on individual, representative windows in order to provide meaningful results for monitoring surface changes and, if necessary, for random quality control of conservation measures. 


\section{ACKNOWLEDGEMENTS}

Our special thanks go to the 'German Federal Environmental Foundation' (DBU) and the 'Deutsch-Polnische Stiftung Kulturpflege und Denkmalschutz' (DPS) in Görlitz, funded by the Federal Government Commissioner for Culture and the Media (BKM), for funding the project. Further thanks to the glass conservators Sławomir Oleszczuk and Katarzyna Wójcik for their great cooperation and warm welcome in the glass restoration workshop in Wroclaw.

Furthermore, we want to thank Ruth Tenschert, John Hindmarch, Leander Pallas, Christoph Altmann and the Centre for Heritage Conservation Studies and Technologies (KDWT) for their support during the project.

\section{REFERENCES}

Akce, D., Remondino, F., Novák, D., Hanusch, T., Schrotter, G., Gruen, A., 2007. Performance Evaluation of a coded structured light system for cultural heritage applications. In: Proc. SPIE 6491, Videometrics IX, 64910V, pp. 1-12, http://doi.org/10.1117/12.705578.

Bellendorf, P., 2007. Metallene Grabplatten aus Franken und Thüringen aus dem 15. bis 18. Jahrhundert - eine interdisziplinäre Studie zum Denkmalbestand und seiner Gefährdung durch Umwelteinflüsse, Bamberg, Univ., Diss., pp. 122-123, https://nbn-resolving.org/urn:nbn:de:bvb:473-opus1368 (14. July 2019).

Brepohl, E., 2013. Theophilus Presbyter und das mittelalterliche Kunsthandwerk, Böhlau Verlag Cologne/ Weimar/ Vienna.

Chen, T., Lensch, H. P. A., Fuchs, C., Seidel, H.-P., 2007. Polarization and Phase-Shifting for 3D Scanning of Translucent Objects. In: Proceedings of IEEE Computer Society Conference on Computer Vision and Pattern Recognition (CVPR) Minneapolis, pp. 1-8.

Díaz-Marín, M. D. C., Aura Castro, E., Sánchez Belenguer, C., Vendrell Vidal, E., 2016. Cyclododecane as opacifier for digitalization of archaeological glass. In: Journal of Cultural Heritage. 17 pp. $131-140$,

https://doi.org/10.1016/j.culher.2015.06.003.

Drewello, R., Kleine, M., Bellendorf, P. 2010. Das mittelalterliche Mosesfenster in Straubing. In: Pilosi, L., Shepard, M. B., Strobl, S. (Eds.), The Art of Collaboration Stained-Glass Conservation in the Twenty-first century, Harvey Miller Publishers, pp.183-192.

Drewello, R. Wetter, N., Rahrig, M., Bellendorf, P. 2010. 3DDokumentation mittelalterlicher Glasmalerei mit der Methode der 3D-Weißlicht-Streifenprojektion. In: Bornschein, F., Torge, M., Sabel, M., Feldmann, I., Kohl, A., Sterzing, N., Hahn, O., Drewello, R., Wetter, N., Rahrig, M., Bellendorf, P. (Eds.) Konservierung mittelalterlicher Glasmalerei im Kontext spezieller materieller und umweltbedingter Gegebenheiten. Dom zu Erfurt St. Marien, Domkapitel, pp. 142-154, https://doi.org/10.5165/hawk-hhg/epublication/43.

Drewello, R., Kleine, M., Bellendorf, P. 2016. Das Mosesfenster in St. Jakob in Straubing im Schnittpunkt von Wissenschaft, Restaurierung und topografischer Dokumentation. In: Corpus Vitrearum Deutschland / Germanisches Nationalmuseum (Eds.), Originale Kaltmalerei auf historischen Glasmalereien, arthistoricum.net, Heidelberg pp. 73-80, https://doi.org/10.11588/arthistoricum.383.545.

Eren, G., 2010. 3D scanning of transparent objects. Université de Bourgogne, 2010DIJOS029, tel-00584061,

https://tel.archives-ouvertes.fr/tel-00584061 (14.July 2019).

Floth, M., Breuer, M., 2011. Optische 3D-Messtechniken für die berührungslose, detaillierte Erfassung von Objektoberflächen in Archäologie und Denkmalpflege. In: Heine, K., Rheidt, K., Henze, F., Riedel, A. (Eds.) Von Handaufmass bis High Tech III, 3D in der historischen Bauforschung, Verlag Philipp von Zabern, Darmstadt/Mainz, pp. 8-13.

Gühring, J., 2002. 3D-Erfassung und Objektrekonstruktion mittels Streifenprojektion, In: Deutsche Geodätische Kommission (Eds.) Reihe C Heft Nr. 560.

Hullin, M.B., Fuchs, M., Ihrke, I., Seidel, H.P., Lensch, H.P.A. 2008. Fluorescent Immersion Range Scanning. In: $A C M$ Transactions on Graphics 27, Issue 3, Article 87, https://doi.org/10.1145/1360612.1360686.

Ihrke, I., Kutulakos, K. N., Lensch, H. P. A., Magnor, M., Heidrich, W., 2010. Transparent and Specular Object Reconstruction. In: Computer Graphics forum, Vol. 29, 8, pp. 2400-2426, https://doi.org/10.1111/j.1467-8659.2010.01753.x.

Mériaudeau, F, Rantoson, R., Fofi, D., Stolz, C., 2012. Review and comparison of non-conventional imaging systems for threedimensional digitization of transparent objects. In: J. Electron. Imag. 21 (2), 021105, pp. 1-6,

https://doi.org/10.1117/1.JEI.21.2.021105.

Rahrig, M., Drewello, R., Lazzeri, A., 2018. Opto-Technical Monitoring - A Standardized Methodology to assess the Treatment of Historical Stone Surfaces. In: Int. Arch. Photogramm. Remote Sens. Spatial Inf. Sci., XLII-2, pp. 945952, https://doi.org/10.5194/isprs-archives-XLII-2-945-2018.

Rantoson, R., Stolz, C., Fofi, D., Mériaudeau, F., 2010. Non Contact 3D Measurement Scheme for Transparent Objects Using UV Structured light. In: 20th International Conference on Pattern Recognition, Istanbul, IEEE, pp. 1646-1649, https://doi.org/10.1109/ICPR.2010.407.

Stylianidis, E., Remondino, F., (Eds.), 2016. 3D Recording, Documentation and Management of Cultural Heritage, Whittles Publishing, Dunbeath.

Salemi, G., Achilli, V., Boatto. G. 2008. 3D Virtual Modelling of a Gothic Stained-Glass Panel. Int. Arch. Photogramm. Remote Sens. Spatial Inf. Sci., Vol. XXXVII, Part B5, pp. $297-$ 302 .

Wedepohl, K. H., 2003. Glas in Antike und Mittelalter. Geschichte eines Werkstoffs, E. Schweizerbart'sche Verlagsbuchhandlung, Stuttgart.

Yeung, S.K., Wu, T.P., Tang, CH. K., Chan, T. F., Osher, S., 2011. Adequate reconstruction of transparent objects on a shoestring budget. CVPR 2011, Colorado Springs, CO, USA, IEEE, pp. 2513-2520. 